\title{
Trends in the prevalence and management of atrial fibrillation in general practice in England and Wales, 1994-1998: analysis of data from the general practice research database
}

\author{
A Majeed, K Moser, K Carroll
}

\begin{abstract}
Objective-To determine the prevalence of atrial fibrillation in England and Wales, and examine trends in its treatment with warfarin and aspirin between 1994 and 1998.

Design-Analysis of data from the general practice research database.

Setting-England and Wales.

Patients-1.4 million patients registered with 211 general practices.

Main outcome measures-Age and sex specific prevalence rates of atrial fibrillation; percentage of patients with atrial fibrillation treated with oral anticoagulants or aspirin.

Results-The prevalence of atrial fibrillation in 1998 was 12.1/1000 in men and 12.7/1000 in women. Prevalence increased from less than $1 / 1000$ in under 35 year olds to over 100/1000 in those aged 85 years and over. There was a $22 \%$ increase in the age standardised prevalence of atrial fibrillation in men and a 14\% increase in women between 1994 and 1998 . The percentage of patients prescribed oral anticoagulants increased from $20 \%$ to $34 \%$ in men and from $17 \%$ to $25 \%$ in women. The percentage of men with atrial fibrillation prescribed aspirin increased from $26 \%$ to $36 \%$, and the percentage of women increased from $24 \%$ to $36 \%$. Applying the age and sex specific prevalence and treatment rates to the population gives an estimate of around 650000 cases of atrial fibrillation in England and Wales. The greatest number of cases occurs in the 75-84 year old age group.
\end{abstract}

Conclusions-The number of patients in the community with identified atrial fibrillation is increasing. There has also been a pronounced increase in the percentage of patients with atrial fibrillation prescribed oral anticoagulants or aspirin.

(Heart 2001;86:284-288)

Keywords: atrial fibrillation; prevalence; treatment; primary care

The risk of stroke is increased three- to sevenfold in patients with non-valvar atrial fibrillation. ${ }^{1-5}$ Because its prevalence increases with age, the proportion of strokes in the population that are caused by atrial fibrillation increases from $1-2 \%$ at age $50-59$ years to around $24 \%$ for those aged $80-89$ years. ${ }^{1}$ Furthermore, strokes associated with atrial fibrillation are usually more severe than strokes from other causes, and more likely to lead to death. ${ }^{6}$ Hence, as the population of the UK ages, the importance of atrial fibrillation as a cause of stroke and subsequent death and disability will increase. $^{7}$

Despite its importance, only a few studies

School of Public Policy, University College London, London WC1H 9EZ, UK

A Majeed

Office for National Statistics, London SW1V 2QQ, UK K Moser

K Carroll

Correspondence to: Dr A Majeed, Room B7-04, Office for National Statistics, 1 Drummond Gate, London SW1V 2QQ, UK azeem.majeed@uclh.org

Accepted 28 February 2001 patients with chronic cardiac disorders is likely to lead to an increase in the prevalence of atrial fibrillation in the UK. For example, the Framingham study in the USA found a threefold increase in the prevalence of atrial fibrillation in men between 1968 and 1989.

Several trials have shown that treatment with oral anticoagulants can reduce the risk of stroke in patients with atrial fibrillation. ${ }^{12} 13$
Aspirin can also reduce the risk of stroke but the reduction in risk is less than with warfarin. In the National service framework on coronary heart disease it is stated that all eligible patients over 60 years of age with atrial fibrillation should be treated with either aspirin or warfarin or both. ${ }^{14}$ However, even among patients who are eligible for treatment, atrial fibrillation is not always treated adequately in primary care. ${ }^{15}$ As with prevalence studies, this finding has often been based on studies carried out in a small group of general practices. There is also very little information on how the treatment of atrial fibrillation is changing over time.

Because of the clinical and public health implications of atrial fibrillation, there is a need for better information on its epidemiology and treatment in primary care. We used information from the general practice research database to examine the prevalence of atrial fibrillation, and its treatment with warfarin and aspirin, in England and Wales between 1994 and 1998.

\section{Methods}

The data for this study came from 211 general practices in England and Wales (total list size 1.4 million) contributing data to the general practice research database. General practices participating in the general practice research 
Table 1 Prevalence and treatment of atrial fibrillation in men in 211 general practices in England and Wales between 1994 and 1998

\begin{tabular}{|c|c|c|c|c|c|c|c|c|}
\hline & \multicolumn{7}{|c|}{ Age (years) } & \multirow[b]{2}{*}{ All ages } \\
\hline & $0-34$ & $35-44$ & $45-54$ & $55-64$ & $65-74$ & $75-84$ & $85+$ & \\
\hline \multicolumn{9}{|l|}{1994} \\
\hline Number with atrial fibrillation & 129 & 119 & 378 & 719 & 1522 & 1450 & 399 & 4716 \\
\hline Prevalence per 1000 & 0.5 & 1.6 & 5.6 & 14.4 & 37.6 & 74.1 & 87.2 & 9.5 \\
\hline \multicolumn{9}{|l|}{ Percentage prescribed oral } \\
\hline Percentage prescribed aspirin & 4.7 & 6.7 & 10.3 & 19.2 & 30.4 & 32.4 & 30.1 & 26.4 \\
\hline \multicolumn{9}{|l|}{1998} \\
\hline Number with atrial fibrillation & 145 & 227 & 557 & 1137 & 2179 & 2332 & 641 & 7218 \\
\hline Prevalence per 1000 & 0.5 & 2.5 & 6.6 & 18.0 & 46.2 & 90.5 & 106.2 & 12.1 \\
\hline \multicolumn{9}{|l|}{ Percentage prescribed oral } \\
\hline anticoagulants & 5.5 & 12.3 & 28.0 & 42.0 & 41.4 & 33.3 & 13.6 & 33.7 \\
\hline Percentage prescribed aspirin & 2.1 & 8.8 & 12.7 & 26.4 & 38.2 & 44.3 & 48.5 & 35.6 \\
\hline
\end{tabular}

database follow agreed guidelines for the recording of clinical and prescribing data, and submit anonymised patient based clinical records to the database at regular intervals. The comprehensiveness and accuracy of the data recorded in the general practice research database have been documented previously and the database has been used extensively for epidemiological research. ${ }^{16}$ All of the 211 practices included in this analysis contributed data which passed quality checks throughout the period 1994-1998. The practices had a combined population with a similar age/sex composition to that of England and Wales. ${ }^{17}$

PREVALENCE OF ATRIAL FIBRILLATION

The diagnosis of atrial fibrillation was based on a clinical diagnosis of atrial fibrillation recorded by a general practitioner on the practice computer system. For each year between 1994 and 1998, we identified all patients who had ever had a diagnosis of atrial fibrillation and extracted information on age, sex, general practice of the patient, and whether the patient had been prescribed an oral anticoagulant or aspirin during the relevant year. We then calculated the prevalence of atrial fibrillation in each age/sex stratum and the percentage of patients with atrial fibrillation prescribed each of these categories of drugs. Finally, age and sex specific prevalence rates were applied to the population of England and Wales in 1998 to estimate the total number of cases of atrial fibrillation in the population.
INTERPRACTICE VARIATION

We examined the interpractice variation in the prevalence and treatment of atrial fibrillation. Because the number of patients with atrial fibrillation in any one practice may be small, data for the period 1994 to 1998 were combined to provide more stable estimates for each practice. For each practice, we calculated the prevalence of atrial fibrillation during this period, and the percentage of patients with atrial fibrillation prescribed each of the categories of drugs examined. We also calculated the proportion of all patients in each practice who were receiving anticoagulation treatment for atrial fibrillation (this gives a measure of the workload generated by treating this group of patients with anticoagulants).

\section{Results}

The prevalence of atrial fibrillation in 1998 was $12.1 / 1000$ in men (table 1 ) and $12.7 / 1000$ in women (table 2). Prevalence rates increased with age from less than 1/1000 in under 35 year olds to over $100 / 1000$ in those aged 85 years and over. Age specific prevalence rates were higher in men than in women in all age groups except the youngest ( $0-34$ years) and the oldest (85 years and over). The prevalence of atrial fibrillation increased between 1994 and 1998 in all age groups in both men and women. Overall, there was a $22 \%$ increase in the age standardised prevalence in men and a $14 \%$ increase in women between 1994 and 1998.

Table 2 Prevalence and treatment of atrial fibrillation in women in 211 general practices in England and Wales between 1994 and 1998

\begin{tabular}{|c|c|c|c|c|c|c|c|c|}
\hline & \multicolumn{7}{|c|}{ Age (years) } & \multirow[b]{2}{*}{ All ages } \\
\hline & $0-34$ & $35-44$ & $45-54$ & $55-64$ & $65-74$ & $75-84$ & $85+$ & \\
\hline \multicolumn{9}{|l|}{1994} \\
\hline Number with atrial fibrillation & 143 & 127 & 233 & 517 & 1351 & 2020 & 1176 & 5567 \\
\hline Prevalence per 1000 & 0.6 & 1.8 & 3.6 & 10.4 & 28.4 & 63.9 & 93.7 & 10.9 \\
\hline \multicolumn{9}{|l|}{ Percentage prescribed oral } \\
\hline anticoagulants & 0.7 & 11.0 & 16.7 & 32.1 & 26.8 & 14.7 & 4.5 & 16.7 \\
\hline Percentage prescribed aspirin & 0.7 & 0.8 & 5.2 & 15.7 & 22.9 & 30.0 & 25.9 & 23.6 \\
\hline \multicolumn{9}{|l|}{1998} \\
\hline Number with atrial fibrillation & 182 & 192 & 366 & 679 & 1759 & 2837 & 1733 & 7748 \\
\hline Prevalence per 1000 & 0.7 & 2.2 & 4.4 & 10.9 & 33.0 & 71.5 & 109.1 & 12.7 \\
\hline \multicolumn{9}{|l|}{ Percentage prescribed oral } \\
\hline anticoagulants & 2.2 & 4.2 & 14.8 & 33.7 & 39.2 & 27.4 & 11.0 & 25.2 \\
\hline Percentage prescribed aspirin & 2.7 & 2.6 & 6.3 & 21.4 & 33.8 & 41.6 & 47.1 & 35.7 \\
\hline
\end{tabular}


Table 3 Atrial fibrillation in England and Wales in 1998

\begin{tabular}{|c|c|c|c|c|c|c|}
\hline \multirow[b]{2}{*}{ Age group (years) } & \multicolumn{2}{|c|}{ Total population } & \multicolumn{2}{|c|}{ Number with atrial fibrillation } & \multicolumn{2}{|c|}{$\begin{array}{l}\text { Number prescribed oral } \\
\text { anticoagulants }\end{array}$} \\
\hline & Men & Women & Men & Women & Men & Women \\
\hline $0-34$ & 12572900 & 11948900 & 6286 & 8364 & 346 & 184 \\
\hline $35-44$ & 3802900 & 3712100 & 9507 & 8167 & 1169 & 343 \\
\hline $45-54$ & 3449400 & 3454500 & 22766 & 15200 & 6374 & 2250 \\
\hline 55-64 & 2569200 & 2630100 & 46246 & 28668 & 19423 & 9661 \\
\hline $65-74$ & 2039600 & 2360800 & 94230 & 77906 & 39011 & 30539 \\
\hline $75-84$ & 1113200 & 1757600 & 100745 & 125668 & 33548 & 34433 \\
\hline $85+$ & 269400 & 747300 & 28610 & 81530 & 3891 & 8968 \\
\hline Total & 25816600 & 26611300 & 308390 & 345504 & 103763 & 86379 \\
\hline
\end{tabular}

Atrial fibrillation is largely a disease of the elderly; in $1998,71 \%$ of cases in men and $82 \%$ of cases in women occurred in those aged 65 years and over.

PRESCRIBING FOR ATRIAL FIBRILLATION

Prescribing of oral anticoagulants in patients with atrial fibrillation increased between 1994 and 1998. The percentage of patients prescribed oral anticoagulants increased from $20 \%$ to $34 \%$ in men (table 1 ), and from $17 \%$ to $25 \%$ in women (table 2). The largest increases in the use of anticoagulants were seen in people aged 75 years and over. The use of oral anticoagulants was greatest among 55-74 year old men, in whom over $40 \%$ of cases were treated with these drugs in 1998. The use of aspirin also increased between 1994 and 1998. The percentage of men with atrial fibrillation prescribed aspirin increased from $26 \%$ to $36 \%$, and the percentage of women increased from $24 \%$ to $36 \%$.

ATRIAL FIBRILLATION IN ENGLAND AND WALES Applying the age and sex specific prevalence and treatment rates to the population gives an estimate of around 650000 cases of atrial fibrillation in England and Wales, of whom around $29 \%$ were treated with anticoagulants in 1998 (table 3).

INTERPRACTICE VARIATION

During the period 1994-1998, the recorded prevalence of atrial fibrillation in the 211 practices varied from $0.7 / 1000$ to $25.2 / 1000$ (table 4). The percentage of patients with atrial fibrillation prescribed anticoagulants varied from around $2 \%$ to $50 \%$, and the percentage prescribed aspirin varied from $8 \%$ to $62 \%$. The overall treatment rate with anticoagulants for patients with atrial fibrillation varied from 0.1/ 1000 patients to $8.9 / 1000$.

\section{Discussion}

Our study is the largest population based study of the epidemiology and treatment of atrial fibrillation carried out in England and Wales.

Table 4 Interpractice variation in prevalence and treatment of atrial fibrillation in 211 general practices in England and Wales, 1994-1998

\begin{tabular}{lllll}
\hline & Mean & $\begin{array}{l}\text { Standard } \\
\text { deviation }\end{array}$ & Minimum & Maximum \\
\hline Prevalence per 1000 & 11.5 & 4.4 & 0.7 & 25.2 \\
$\begin{array}{l}\text { Percentage prescribed oral } \\
\quad \text { anticoagulants }\end{array}$ & 23.6 & 8.6 & 1.8 & 50.0 \\
$\begin{array}{l}\text { Percentage prescribed aspirin } \\
\text { Number treated with anticoagulants }\end{array}$ & 30.1 & 8.0 & 8.1 & 61.6 \\
$\quad$ per 1000 registered patients & 2.7 & 1.4 & 0.1 & 8.9 \\
\hline
\end{tabular}

The prevalence of atrial fibrillation of just over $1 \%$ is consistent with that found by other researchers. We confirm that atrial fibrillation is largely a disease of the elderly, with the great majority of the cases occurring in people aged 65 years and over. There was a pronounced increase in the age standardised prevalence of atrial fibrillation between 1994 and 1998. This is probably a result of several factors, including greater awareness of the risks associated with atrial fibrillation, improved diagnosis, and a true increase in prevalence owing to an increased survival of people with conditions such as ischaemic heart disease that predispose to atrial fibrillation. There was also an improvement in the management of atrial fibrillation in primary care between 1994 and 1998, with an increased percentage of patients prescribed oral anticoagulants and aspirin.

STRENGTHS AND WEAKNESSES OF STUDY

This study used information from the general practice research database, a large and well validated general practice derived database. Several previous validation studies have confirmed the accuracy of the clinical data recorded in it. Because of the large number of cases included, our study provides reliable estimates of the prevalence and treatment of atrial fibrillation by age group and sex. We were also able to examine time trends in the treatment of atrial fibrillation.

The main weakness of the study is that we did not validate the diagnosis of atrial fibrillation-for example, by examining whether patients had their diagnosis confirmed by ECG. The study also only used information on diagnosed cases of atrial fibrillation and hence asymptomatic cases who had not come to the attention of their general practitioner would not be included. In the cardiovascular heart study, about $12 \%$ of cases of atrial fibrillation were identified through ECG screening. ${ }^{18}$ Similarly, Wheeldon and colleagues found that of 65 elderly patients with atrial fibrillation, five $(8 \%)$ were first identified by ECG screening. ${ }^{10}$

There may also be deficiencies in the prescribing data. For example, some patients with atrial fibrillation may have their anticoagulant monitoring and prescribing carried out entirely in secondary care. However, as most patients will have their treatment prescribed by their general practitioner, the effect of this is not likely to be great. ${ }^{19}$ Some patients may also buy their aspirin over the counter rather than have it prescribed by their doctor. Again, the 
effect of this is not likely to be large, particularly among the older age groups, who are eligible for free prescriptions. Finally, the practices in the general practice research database are volunteers and so may not be a typical sample of general practices in England and Wales.

COMPARISON WITH PREVIOUS STUDIES

The prevalence rate of atrial fibrillation of around $1 \%$ in this study is consistent with the findings of previous epidemiological studies. The overall rate of treatment with anticoagulants in patients with atrial fibrillation is higher than the $23 \%$ found by Sudlow and colleagues in an earlier study from the north of England ${ }^{8}$ and similar to the $31 \%$ reported by Kalra and associates. ${ }^{20} \mathrm{~A}$ substantially higher treatment rate of $55 \%$ was reported in a study of patients registered with the Kaiser Permananente medical care programme in northern California. ${ }^{21}$ However, general practices in the UK register patients from all sections of the population, unlike US health maintenance organisations, which typically do not include patients from lower socioeconomic groups.

IMPLICATIONS OF THE STUDY

The use of anticoagulants and aspirin among people with atrial fibrillation increased between 1994 and 1998, with the largest increase occurring among the elderly. However, treatment rates remain relatively low, suggesting there is still scope for further improvement. Because atrial fibrillation is common, the population benefits of treatment in terms of reduced numbers of thromboembolic strokes are potentially large and outweigh the risks, such as an increased risk of haemorrhagic strokes.

Our study suggests that there about 650000 patients with atrial fibrillation in England and Wales, of whom $29 \%$ received anticoagulants in 1998. An overview of the benefits of anticoagulation treatment suggested that treating 1000 patients with atrial fibrillation with oral anticoagulants would prevent about 30 strokes and seven deaths every year, at the cost of seven major bleeding episodes. ${ }^{22}$ This suggests that current treatment rates with anticoagulants are preventing about 5700 strokes and 1330 deaths every year in England and Wales, at a cost of 1330 major bleeding episodes. Sudlow and colleagues suggested that around $40-60 \%$ of patients with atrial fibrillation are eligible for treatment with anticoagulants. ${ }^{8}$ Hence, between 72000 and 202000 additional patients with atrial fibrillation in England and Wales may be eligible for anticoagulation treatment. Treating this number of extra patients would substantially increase the workload of primary health care teams, cardiologists, stroke specialists, and anticoagulation clinics. ${ }^{23}$

In the National service framework for coronary heart disease it is stated that general practices should be able to identify the proportion of their patients over 60 years of age with atrial fibrillation prescribed warfarin or aspirin. Our study provides baseline figures for the expected prevalence of atrial fibrillation in primary care and the percentage of patients currently prescribed aspirin or anticoagulants. The study also shows that some practices are much more effective than others in treating patients who have atrial fibrillation. Further examination of how these practices identify and provide care for this group of patients may reveal lessons that can be applied more widely among general practices in the National Health Service. Some practices were treating around nine patients per 1000 total list size with oral anticoagulants (about 18 patients per general practitioner and around 90 patients in a 10000 patient practice), compared with less than one per 1000 in some other practices. Hence, the workload generated by treating patients with atrial fibrillation with oral anticoagulants varied widely between general practices.

UNANSWERED QUESTIONS AND FUTURE RESEARCH Some important questions about atrial fibrillation remain unanswered. For example, what proportion of the increase in the prevalence of atrial fibrillation is real and what proportion reflects better awareness and detection of the condition? Better information is also needed on how factors such as comorbidity influence the likelihood of treatment, and the association between treatment, comorbidity, and patient outcomes. Some of these questions will be investigated in future studies using the general practice research database.

This study was funded by the Office for National Statistics. AM holds a national primary care career scientist award and is funded by the NHS Research and Development Directorate. We thank the Morbidity and Health Care Research Team at the thank the Morbidity and Health Care Research Team at the
Office for National Statistics for their help and the Medicines Office for National Statistics for their help and the Medicines
Control Agency for permission to use data from the GPRD.

1 Wolf PA, Abbott RD, Kannel WB. Atrial fibrillation as an independent risk factor for stroke: The Framingham study. Stroke 1991;22:983-8.

2 Wolf PA, Abbott RD, Kannel WB. Atrial fibrillation: a major Wolf PA, Abbott RD, Kannel WB. Atrial fibrillation: a major
contributor to stroke in the elderly. The Framingham contributor to stroke in the elderly. The
study. Arch Intern Med 1987;147:1561-4.

3 Cerebral Embolism Task Force. Cardiogenic brain embolism: the second report of the Cerebral Embolism Task Force. Arch Neurol 1989;46:727-43.

4 Atrial Fibrillation Investigators. Risk factors for stroke and efficacy of antithrombotic therapy in atrial fibrillation. Arch Intern Med 1994;154:1449-57.

5 Roy D, Marchand E, Gagne P, et al. Usefulness of anticoagulant therapy in the prevention of embolic complications of atrial fibrillation. Am Heart f 1986;112:1139-43.

6 Lin HJ, Wolf PA, Kelly-Hayes M, et al. Stroke severity in atrial fibrillation: the Framingham Study. Stroke 1996;10: 1765-9.

7 Carroll K, Majeed A. Trends in mortality and hospital admissions associated with atrial fibrillation in England and Wales. Health Stat $O 2001 ; 9: 37-43$.

8 Sudlow M, Thomson R, Thwaites B, et al. Prevalence of atrial fibrillation and eligibility for anticoagulants in the atrial fibrillation and eligibility for an

9 Lip GYH, Golding DJ, Nazir M, et al. A survey of atrial fibrillation in general practice: the West Birmingham atrial fibrillation project. Br f Gen Pract 1997; 47:285-9.

10 Wheeldon NM, Tayler DI, Anagnostou E, et al. Screening for atrial fibrillation in primary care. Heart 1998;79:50-5.

11 Hill JD, Mottram EM, Killeen PD. Study of the prevalence of atrial fibrillation in general practice patients over 65 years of age. $\mathcal{F}$ R Coll Gen Pract 1987;37:172-3.

12 Hart RG, Benavente O, McBride R, et al. Antithrombotic therapy to prevent stroke in patients with atrial fibrillation: a meta-analysis. Ann Intern Med 1999;131:492-501.

13 Hart RG, Pearce IA, Rothbart RM, et al Stroke with intermittent atrial fibrillation: incidence and predictors during mittent atrial fibrillation: incidence and predictors during aspirin therapy. Stroke prevention in atrial
investigators. F Am Coll Cardiol 2000;35:183-7.

14 Department of Health. National service framework for coronary heart disease. London: Department of Health, 2000. 
15 Sudlow CM, Rodgers H, Kenny RA, et al. Service provision and use of anticoagulants in atrial fibrillation. $B M F$ 8-60.

16 Hollowell J. The general practice research database: quality of morbidity data. Pop Trends 1997;87:36-40.

17 Office for National Statistics. Key health statistics from general practice 1998. (Series MB6 No 2.) London: Office for National Statistics, 2000.

18 Furberg CD, Psaty BM, Manolio TA, et al, for the CHS Collaborative Research group. Prevalence of atrial fibrillation in elderly subjects (the cardiovascular health study). Am F Cardiol 1994;74:236-41.

19 Majeed A, Evans N, Head P. What can PACT tell us about prescribing in general practice? $B M F$ 1997;315:1515-19.
20 Kalra L, Yu G, Perez I, et al. Prospective cohort study to determine if trial efficacy of anticoagulation for stroke prevention in atrial fibrillation translates into clinical effectiveness. BMF 2000;320:1236-9.

21 Go AS, Hylek EM, Borowsky LH, et al. Warfarin use among ambulatory patients with nonvalvular atrial fibrillation: the anticoagulation and risk factors in atrial fibrillation

22 Turazza FM, Franzosi MG. Is anticoagulation therapy underused in elderly patients with atrial fibrillation? Drugs Aging 1997;10:174-84.

23 Ezekowitz MD. Atrial fibrillation: the epidemic of the new millennium. Ann Intern Med 1999;131:492-501.

\section{Coronary artery embolism complicating heart valve surgery: role of mechanical thrombectomy}

An 82 year old woman underwent mitral valve annuloplasty and aortic valve replacement with a bioprosthesis. Sudden pronounced ST elevation was recorded on cardiac monitor while she was still intubated and sedated within 24 hours of the operation. Preoperative coronary angiogram was normal. Immediate cardiac catheterisation showed mid left anterior descending artery (LAD) embolic occlusion with TIMI 0 flow (panel A). Using an $8 \mathrm{~F}$ XB3.5 guiding catheter, the occlusion was crossed with a 0.014 inch guidewire. Mechanical thrombectomy was performed using the Possis angiojet rheolytic thrombectomy system (panel B) The system consists of a 5 French angiojet catheter, a pump set, and a drive unit. The pump set, powered by the drive unit, delivers pressurised saline to the catheter. Powerful, high velocity saline jets create suction current at the catheter tip resulting in rapid mechanical thrombolysis and removal via the catheter's effluent lumen. The pro-arrhythmic venturi effect produced by the system necessitates the use of a temporary pacing wire. Adjunctive balloon angioplasty and stenting was performed. TIMI 3 flow was restored. The final angiogram is shown in panel C. This case highlights the niche role of mechanical thrombectomy devices in thrombo-occlusive coronary artery disease when both thrombolytics and glycoprotein IIb/ IIIa receptor antagonists are contraindicated.

ON-HING KWOK CAMPBELL ROGERS vohkwok@netvigator.com
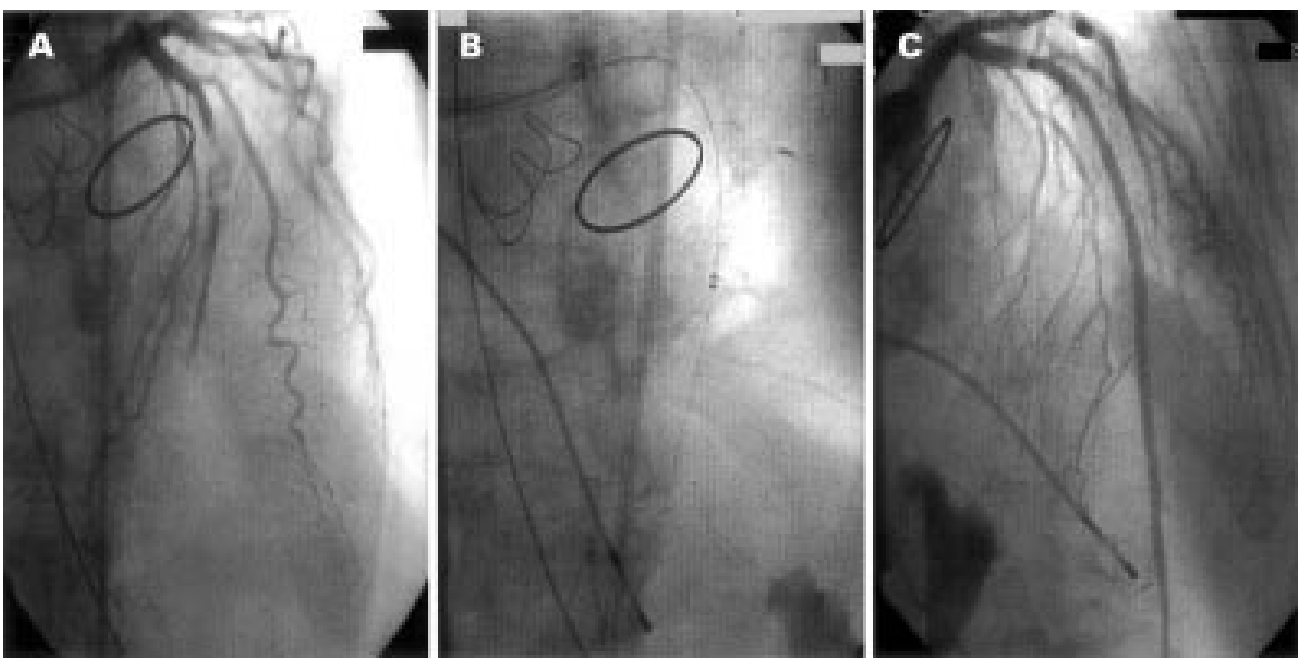